\title{
Color feature extraction of HER2 Score 2+ overexpression on breast cancer using Image Processing
}

\author{
Izzati Muhimmah ${ }^{1, *}$, Dadang Heksaputra ${ }^{2}$, and Indrayanti ${ }^{3}$ \\ ${ }^{1}$ Master of Informatics Engineering Universitas Islam Indonesia, Indonesia \\ ${ }^{2}$ Master of Informatics Engineering Universitas Islam Indonesia, Indonesia \\ ${ }^{3}$ Faculty of Medicine Universitas Muhammadiyah Yogyakarta, Indonesia
}

\begin{abstract}
One of the major challenges in the development of early diagnosis to assess HER2 status is recognized in the form of Gold Standard. The accuracy, validity and refraction of the Gold Standard HER2 methods are widely used in laboratory (Perez, et al., 2014). Method determining the status of HER2 (human epidermal growth factor receptor 2) is affected by reproductive problems and not reliable in predicting the benefit from anti-HER2 therapy (Nuciforo, et al., 2016). We extracted color features by methods adopting Statistics-based segmentation using a continuous-scale naïve Bayes approach. In this study, there were three parts of the main groups, namely image acquisition, image segmentation, and image testing. The stages of image acquisition consisted of image data collection and color deconvolution. The stages of image segmentation consisted of color features, classifier training, classifier prediction, and skeletonization. The stages of image testing were image testing, expert validation, and expert validation results. Area segmentation of the membrane is false positive and false negative. False positive and false negative from area are called the area of system failure. The failure of the system can be validated by experts that the results of segmentation region is not membrane HER2 (noise) and the segmentation of the cytoplasm region. The average from 40 data of HER 2 score $2+$ membrane images show that $75.13 \%$ of the area is successfully recognized by the system.
\end{abstract}

\section{Introduction}

The accuracy of the status Human Epidermal Growth Factor Receptor 2 (HER2) is very important to optimize the result of breast cancer treatmementPerez, et al., 2014). Human epidermal growth factor receptor 2 (HER2) overexpression in breast cancer is an indicator of poor prognosis. HER2 is a prerequisite for a treatment with tissue targeting the epidermal growth receptor (Cresti, et al., 2016). One of the major challenges in the development of early diagnosis to assess HER2 status is recognized in the form of Gold Standard. The accuracy, validity and refraction of the Gold Standard HER2 methods are widely used in laboratory (Perez, et al., 2014). Method determining the status of HER2 (human epidermal growth factor receptor 2) is affected by reproductive problems and not reliable in predicting the benefit from anti-HER2 therapy (Nuciforo, et al., 2016).

The computer-assisted research is carried out to improve the accuracy and reproducibility of breast tissue assessment. Breast tissue specimen image assessment has been done in recent years. Brugmann, et al. (2012) developed and validated a software which is capable of
IHC scoring algorithms automatically based on membrane connectivity on each image. Keller, Chen, and Gavrielides (2012) developed a system which classifies IHC score based on color feature. The system is built using algorithms fuzzy c-means clustering in the HSV color space. Hall et al. (2008) resulted in a system which is able to give a score of IHC using multiple stages, such as selection and image capturing, color decomposition, and membrane isolation algorithm (MIA) with three features based on the average intensity of membrane area. Masmoudi et al. (2009) developed an automated assessment method of HER2 immunohistochemistry. Labellapansa, Muhimmah, and Indrayanti (2013) performed an image analysis by providing a classification HER2 score 1+ and HER2 score $3+$ using Otsu's thresholding segmentation method.

The state of the art from the research domain in table 1 shows the gap to conduct this research. We extracted color features by methods adopting Statistics-based segmentation using a continuous-scale naïve Bayes approach.

\footnotetext{
*Corresponding author: izzati@uii.ac.id
} 
Table 1. State of the art

\begin{tabular}{|c|c|c|c|c|c|c|c|c|c|c|c|c|c|c|c|}
\hline \multirow[b]{2}{*}{ No } & \multirow[b]{2}{*}{ Name } & \multicolumn{14}{|c|}{ Methods } \\
\hline & & 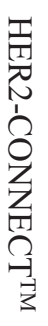 & 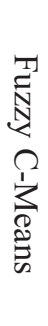 & 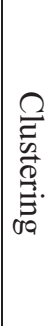 & 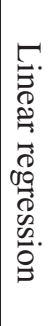 & 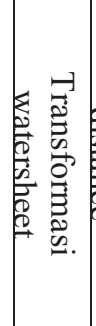 & 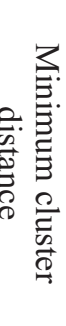 & $\begin{array}{l}T \\
0 \\
0 \\
0.0 \\
0 \\
0 \\
0 \\
0 \\
0 \\
0 \\
0 \\
0 \\
0 \\
0 \\
0\end{array}$ & 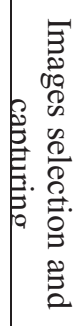 & 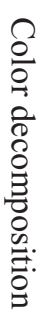 & $\begin{array}{l}\sigma \\
0 \\
0 \\
\vdots \\
0 \\
\infty \\
\Xi \\
0 \\
0 \\
0 \\
0\end{array}$ & 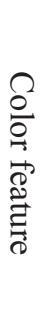 & 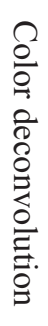 & 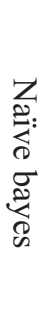 & 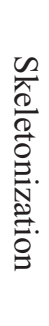 \\
\hline 1 & Brügmann, et al (2012) & $\sqrt{ }$ & & & & & & & & & & & & & \\
\hline 2 & Keller, Chen, and Gavrielides (2012) & & $\sqrt{ }$ & $\sqrt{ }$ & & & & & & & & & & & \\
\hline 3 & Masmoudi, et al (2009) & & & & $\sqrt{ }$ & $\sqrt{ }$ & $\sqrt{ }$ & $\sqrt{ }$ & & & & & & & \\
\hline 4 & Hall, et al (2008) & & & & & & & & $\sqrt{ }$ & $\sqrt{ }$ & & & & & \\
\hline 5 & $\begin{array}{l}\text { Labellapansa, Muhimmah, and } \\
\text { Indrayanti (2013) }\end{array}$ & & & & & & & & & & $\sqrt{ }$ & & & & \\
\hline 6 & Laursen, et al (2014) & & & & & & & & & & & $\sqrt{ }$ & & $\sqrt{ }$ & \\
\hline 7 & proposed & & & & & & & & & & & $\sqrt{ }$ & $\sqrt{ }$ & $\sqrt{ }$ & $\sqrt{ }$ \\
\hline
\end{tabular}

\section{Methods}

\section{A. Color Deconvolution}

Color deconvolution is needed in the application of membrane extraction method. The approach has been used in previous of this method to divide the allegations of cell membrane overexpression. Ruifrok and Johnston (2001) gave OD matrix for IHC staining H, E and DAB. Color decomposition was applied to the original image and resulted in grayscale and image with DAB stain with hematoxylin stain (Hall, et al., 2008).

\section{B. Calculation of Color Feature}

The pixel values were obtained from the clustering using color deconvolution method. The pixel values were used as criteria for location of the position coordinates. RGB values of the layer were taken to apply the calculation of color features. The chromaticities $(\mathrm{r}, \mathrm{g}, \mathrm{b})$ were given by the raw color value divided with the sum of all the color total value (Laursen, et al., 2014).

$$
(r, g, b)^{T}=\frac{(R, G, B)^{T}}{R+G+B}
$$

There were also excess green (ExG) and excess red (ExR) (Meyer and Neto, 2008).

$$
\begin{aligned}
\mathrm{ExG} & =2 . \mathrm{G}-\mathrm{R}-\mathrm{B} \\
\mathrm{ExR} & =1.4 \mathrm{R}-\mathrm{G}
\end{aligned}
$$

\section{Classifier Training}

Flexible naïve Bayes was used in this section (Perez, Larranaga, and Inza, 2009). The classifier training consisted of estimating the probability density function for each object and background color feature. The probability density was determined from the annotated images. Numerical vectors were created, containing the values of the four raw colors, namely chromaticities, ExG and ExR values of each pixel. The probability function $\left[\mathrm{P}\left(\mathrm{F}_{\mathrm{k}}=\mathrm{x} \mid \mathbb{C}\right)\right]$ for each of the $\mathrm{k}$ was given input variables conditional on class $\mathbb{C}$. Kernel density estimation with a Gaussian kernel $\mathrm{K}(\mathrm{x})$ has a bandwidth $\mathrm{h}$ (Silverman, 1986), where $\mathrm{L}$ is the number of observations (Laursen, et al., 2014).

$$
\begin{gathered}
\mathrm{P}\left(\mathrm{F}_{\mathrm{k}}=\mathrm{x} \mid \mathbb{C}\right)=\frac{1}{\mathrm{~L} \cdot \mathrm{h}} \sum_{\mathrm{i}-1}^{\mathrm{L}} \mathrm{K}\left(\frac{\mathrm{x}-\mathrm{x}_{\mathrm{i}}}{\mathrm{h}}\right) \\
\mathrm{K}(\mathrm{x})=\frac{1}{\sqrt{2 \pi}} \mathrm{e}^{\frac{-\mathrm{x}^{\mathrm{x}}}{\mathrm{x}}}
\end{gathered}
$$

\section{Classifier Prediction}

The color feature extraction is based on the criteria diagnosed by a physician as HER2 membrane. $(\$)$ is a group of background and (V) is the object. Support groups $\mathbb{C}$ are symbolized by $\mathbf{P}(\mathbb{C}, \vec{F})$, where the vector $\vec{F}$ is a vector of $F_{1}, \ldots, F_{M}$ features $M . P(\mathbb{C})$ is the previous probability of group $\mathbb{C}$.

$$
\begin{aligned}
& \mathrm{P}(\mathbb{C}, \overrightarrow{\mathrm{F}})=\mathrm{P}(\mathbb{C}) \cdot \mathrm{P}\left(\mathrm{F}_{1} \mid \mathbb{C}\right) \cdot \mathrm{P}\left(\mathrm{F}_{2} \mid \mathrm{F}_{1}, \mathbb{C}\right) \cdot \mathrm{P}\left(\mathrm{F}_{3} \mid \mathrm{F}_{1}, \mathrm{~F}_{2}, \mathbb{C}\right)
\end{aligned}
$$

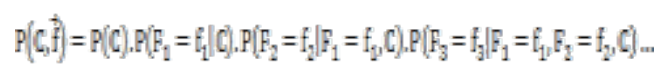

Characteristic assumptions of color features include probability functions. It can be written to equation (10).

$$
\mathrm{P}(\mathbb{C}, \overrightarrow{\mathrm{F}}) \simeq \prod_{\mathrm{m}=1}^{\mathrm{M}} \mathrm{P}\left(\mathrm{F}_{\mathrm{k}} \mid \mathbb{C}\right)
$$

Feature input is denoted as $F_{m}$, where $\mathrm{m} \in\{1, \ldots, \mathrm{m}\}$. Naïve Bayes classifier is chosen 
because it provides values in predicting the dependent input, and determining the increase in the ratio of the input that is independent (Hand dan $\mathrm{Yu}, 2001)$. A pixel is classified as an object if $\mathrm{P}(\mathbb{V} \mid \overrightarrow{\mathrm{F}}) \geq \mathrm{P}(\mathbb{S}, \overrightarrow{\mathrm{F}})$ and if not then in the background (Laursen, et al., 2014).

$$
\mathrm{P}(\mathrm{V} \mid \overrightarrow{\mathrm{F}})=\frac{\mathrm{P}\left(\mathrm{V}_{a} \overrightarrow{\mathrm{F}}\right)}{\mathrm{P}\left(\mathrm{V}_{2} \overrightarrow{\mathrm{F}}\right)+\mathrm{P}(\mathrm{S}, \overrightarrow{\mathrm{F}})}
$$

\section{E. Skeletonization}

The extraction results of the membrane skeletonization technique were used to obtain a linear line to shape the object of the membrane extraction model. Skeleton object is an important topological description of the object of two-dimensional binary image. It has been used in a wide range of applications (Levine, 1985; Serra, 1982; Pitas \& Venetanopoulos, 1990). The skeleton formula of Serra (1982) can be seen in equation (12).

$$
\mathrm{SK}(\mathrm{X})=\mathrm{U}_{\mathrm{n}=0}^{\mathrm{N}} \mathrm{S}_{\mathrm{n}}(\mathrm{X})=\mathrm{U}_{\mathrm{n}=0}^{\mathrm{N}}\left[\left(\mathrm{X} \ominus \mathrm{nB}^{\mathrm{s}}\right)-\left(\mathrm{X} \ominus \mathrm{nB}^{\mathrm{s}}\right)_{\mathrm{B}}\right](
$$

where Sn (X) shows a part of skeleton SK (X) corresponding to the maximum radius in disk.

\section{Research Methodology}

We extracted color features by methods adopting Statistics-based segmentation using a continuous-scale naïve Bayes approach. In this study, there were three parts of the main groups, namely image acquisition, image segmentation, and image testing. The stages of image acquisition were image data collection and color deconvolution. The stages of image segmentation consisted of color features, classifier training, classifier prediction, and skeletonization. The stages of image testing were image testing, expert validation, and expert validation results (see fig. 1).

The datasets of HER2 images were obtained from the Histology Laboratory of Pathology, Universitas Muhammadiyah Yogyakarta. The tool was sigma hd microscope camera, olympus. The images were taken from patient preparation with a microscope at 400x magnification. The staining of HER2 was done by using IHC which was less expensive than FISH and $\mathrm{CISH}$ (Dobson, et al., 2010). The datasets used 40 images. The data were a diagnosis of HER2 score $2+$. The color deconvolution was the automatic annotation to separate the alleged membranes and cells in the image. The results of this annotation were used as criteria to object and background color feature. The calculation of color features was done by using equation (1), equation (2), and equation (3). The calculation values of the color features were processed by classifier training and classifier prediction. They were the eight-layer image namely red (R), green $(\mathrm{G})$, blue $(B)$, excess green (ExG), Chromaticities red (r), Chromaticities green (g), Chromaticities blue (b), and excess red (EXR). The results of classifier training and classifier prediction were to perform skeletonization. The skeletonization was done by experts to justify the overexpression of the membrane criteria.
The researchers used the approach to the average value of the threshold pixel in the image to provide a solution to the justification of experts. The results of the final image underwent the validation process by experts to provide justification to determine the percentage of successful segmented membrane by the system model.

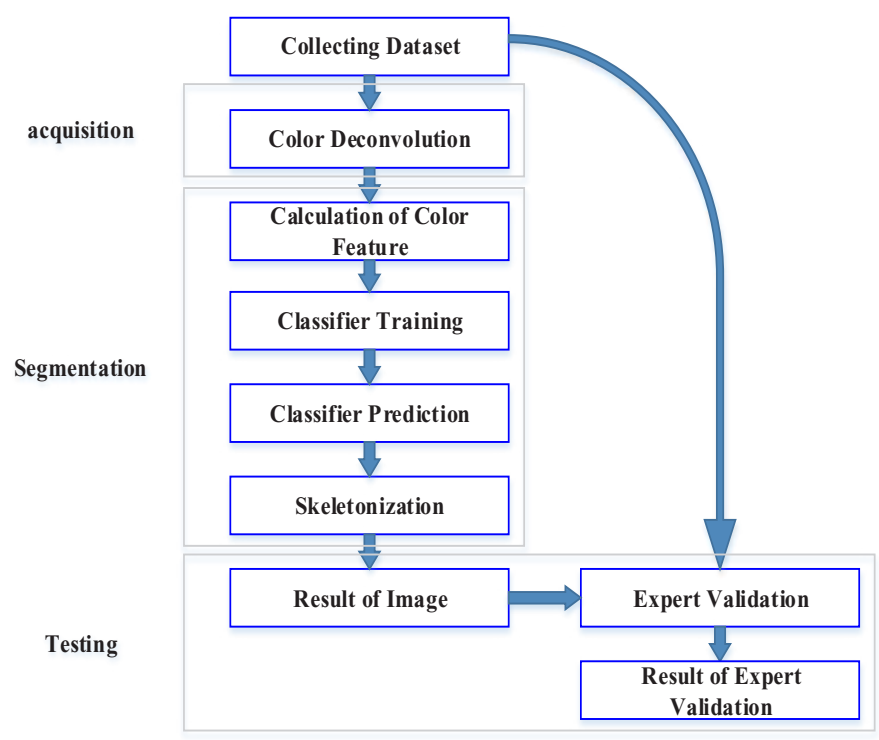

Fig 1. Research process

\section{Results}

This section shows the results of the proposed method. Figure 2 is an example of the implementation of the system model. The system model implementation was designed from the datasets. Ten samples from the image datasets were taken to the modeling system. The end results were validated by physicians. The validation was intended to determine the accuracy of the percentage rate by the membrane systems model. Figure 2 (a) is the original image without image processing. The initial image was the result of the image on hd microscope sigma camera, olympus. Figure 2 (b) shows the results of the color deconvolution, calculation of color features, classifier training, and classifier prediction. Figure 2 (c) is the result of the image skeletonization and application. Figure 2 (d) is the final image. The final image was validated by experts to provide an assessment of the membrane percentage. It is segmented by the system model.

Membrane features contain microscopic images of breast cancer cells using stain Immunuhistochemistry/IHC. They are contained on the distribution of RGB image layer. Sample distribution histogram value of the RGB layer on the membrane characteristics can be seen in figure 3 . The $\mathrm{x}$-axis is the range of variations in the intensity image pixel value ranging from 0 to 255 (grayscale). The y-axis represents the sum of the number of pixels contained in the image. Figure 3 is a sample of the RGB histogram distribution on HER2 score $2+$. The results are compared with the variation in the intensity of membrane staining positive control specimen. The results of the distribution, HER2 score $2+$ membrane values are in the middle of the range 
of values ranging from 100 to 200 of variations in the intensity image pixel values.

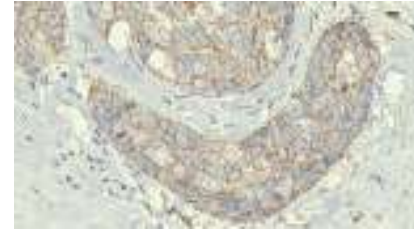

(a)

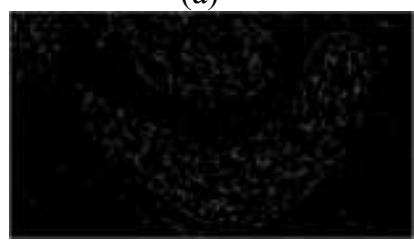

(c)

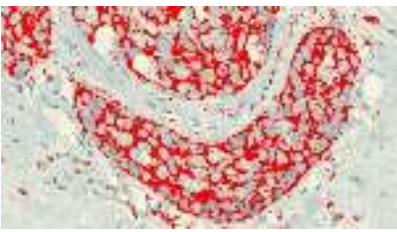

(b)

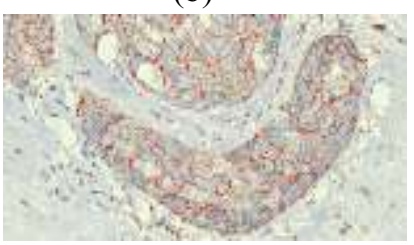

(d)
Fig 2. Example of the stages of the system model implementation. (a) Original image. (b) Image of the alleged membranes by color feature extraction. (c) Binary image skeletonization result. (d) Final result.

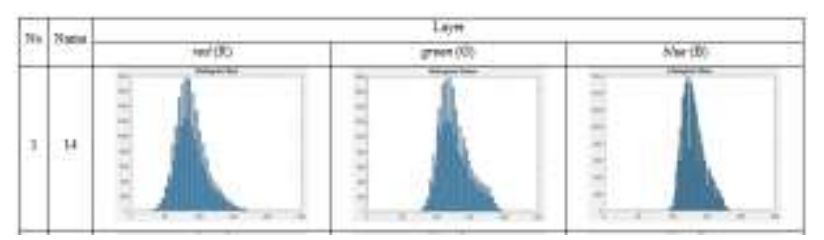

Fig 3. Histogram distribution sample on HER2 score 2+

\section{Testing}

The result testing showed the accuracy of the system model which was designed and implemented. The percentage value was derived from physician assessment of anatomic pathology (Table 2).

Table 2. Results of validation HER2 Score $2+$ by physician

\begin{tabular}{|c|c|c|c|c|c|c|}
\hline \multirow{2}{*}{ No } & \multirow{2}{*}{ File } & \multicolumn{5}{|c|}{ Level of success } \\
\hline & Name & $60 \%$ & $70 \%$ & $80 \%$ & $90 \%$ & $95 \%$ \\
\hline $\mathbf{1}$ & 14 & $\sqrt{ }$ & & & & \\
\hline $\mathbf{2}$ & 15 & & $\sqrt{ }$ & & & \\
\hline $\mathbf{3}$ & 16 & & & $\sqrt{ }$ & & \\
\hline $\mathbf{4}$ & 17 & & & & $\sqrt{ }$ & \\
\hline $\mathbf{5}$ & 18 & & & & $\sqrt{ }$ & \\
\hline $\mathbf{6}$ & 19 & & & & & $\sqrt{ }$ \\
\hline $\mathbf{7}$ & 20 & & & & & $\sqrt{ }$ \\
\hline $\mathbf{8}$ & 26 & & & $\sqrt{ }$ & & \\
\hline $\mathbf{9}$ & 27 & & & & $\sqrt{ }$ & \\
\hline $\mathbf{1 0}$ & 28 & & & & $\sqrt{ }$ & \\
\hline $\mathbf{1 1}$ & 29 & & $\sqrt{ }$ & & & \\
\hline $\mathbf{1 2}$ & 30 & & & & & $\sqrt{ }$ \\
\hline $\mathbf{1 3}$ & 31 & $\sqrt{ }$ & & & & \\
\hline $\mathbf{1 4}$ & 32 & $\sqrt{ }$ & & & & \\
\hline $\mathbf{1 5}$ & 33 & & $\sqrt{ }$ & & & \\
\hline $\mathbf{1 6}$ & 34 & & $\sqrt{ }$ & & & \\
\hline $\mathbf{1 7}$ & 35 & & $\sqrt{ }$ & & & \\
\hline $\mathbf{1 8}$ & 45 & $\sqrt{ }$ & & & & \\
\hline $\mathbf{1 9}$ & 46 & $\sqrt{ }$ & & & & \\
\hline $\mathbf{2 0}$ & 48 & & & $\sqrt{ }$ & & \\
\hline $\mathbf{2 1}$ & 49 & & $\sqrt{ }$ & & & \\
\hline $\mathbf{2 2}$ & 50 & & & $\sqrt{ }$ & & \\
\hline $\mathbf{2 3}$ & 60 & & $\sqrt{ }$ & & & \\
\hline
\end{tabular}

\begin{tabular}{|l|l|l|l|l|l|l|}
\hline $\mathbf{2 4}$ & 62 & & $\sqrt{ }$ & & & \\
\hline $\mathbf{2 5}$ & 66 & & $\sqrt{ }$ & & & \\
\hline $\mathbf{2 6}$ & 67 & $\sqrt{ }$ & & & & \\
\hline $\mathbf{2 7}$ & 68 & $\sqrt{ }$ & & & & \\
\hline $\mathbf{2 8}$ & 87 & & $\sqrt{ }$ & & & \\
\hline $\mathbf{2 9}$ & 88 & & $\sqrt{ }$ & & & \\
\hline $\mathbf{3 0}$ & 96 & & & $\sqrt{ }$ & & \\
\hline $\mathbf{3 1}$ & 97 & & & $\sqrt{ }$ & & \\
\hline $\mathbf{3 2}$ & 98 & & & $\sqrt{ }$ & & \\
\hline $\mathbf{3 3}$ & 102 & & & $\sqrt{ }$ & & \\
\hline $\mathbf{3 4}$ & 103 & & & $\sqrt{ }$ & & \\
\hline $\mathbf{3 5}$ & 104 & & & $\sqrt{ }$ & & \\
\hline $\mathbf{3 6}$ & 105 & & $\sqrt{ }$ & & & \\
\hline $\mathbf{3 7}$ & 106 & & $\sqrt{ }$ & & & \\
\hline $\mathbf{3 8}$ & 107 & & $\sqrt{ }$ & & & \\
\hline $\mathbf{3 9}$ & 117 & & $\sqrt{ }$ & & & \\
\hline $\mathbf{4 0}$ & 119 & & & & $\sqrt{ }$ & \\
\hline
\end{tabular}

Segmentation of the membrane can be false positive and false negative. Regional false positive and false negative are called to the area of system failure. The failure of the system can be validated by experts to have the results.

a. Segmentation of the areas is not membrane HER2 (noise).

b. Segmentation shows cytoplasmic region.

The average of the images in all the datasets shows membrane HER2 score 2+. $75.13 \%$ of the area is successfully recognized by the system.

\section{Conclusion}

Based on the discussion and testing of the system, it can be concluded that:

1. The results of the distribution, HER2 score 2+ membrane values are in the middle of the range of values ranging from 100 to 200 of variations in the intensity image pixel values.

2. The algorithm to extract membrane of breast cancer cells with image processing has been shown successfully to identify membrane in HER2 score $2+$.

A research grant from Hibah PUPT from Ministry RISTEKDIKTI of Indonesia for the period of 2015-2016, contract no. 001/HB-LIT/III/2015, is gratefully acknowledged.

\section{References}

1. Brügmann, A., et al. (2012). Digital image analysis of membrane connectivity is a robust measure of HER2 immunostains. Breast Cancer Res Treat, 132:41-49.

2. Dobson, L., et al. (2010). Image analysis as an adjunct to manual HER-2 immunohistochemical review : a diagnostic tool to standardize interpretation. Blackwell Publishing Ltd, Histopathology , 57, 27-38.

3. Hall, B. H., et al. (2008). Computer-assisted assessment of the Human Epidermal Growth Factor Receptor 2 immunohistochemical assay in imaged histologic sections using a membrane isolation algorithm and quantitative analysis of positive 
controls. BMC Medical Imaging, 1-13.

4. Hand, D. and Yu, K. (2001). Idiot's Bayes : Not to Stupid after All. int. stat. Rev. 69, 385-398.

5. Labellapansa, A., Muhimmah, I., and Indrayanti. (2013). Klasifikasi Citra Immunohistokimia Sel Kanker Payudara HER2 Skore 1+ dan 3+. Seminar Nasional Informatika Medis (SNIMed), 24.

6. Laursen, M. S., et al. (2014). Statistics-based Segmentation using a Continuos Scale Naive Bayes Approch. Elsevier Computer in Electronics in Algiculture, 271-277.

7. Levine, M. D. (1985). Vision in Main and Machine. McGraw-Hill.

8. Masmoudi, H., et al. (2009). Automated quantitative assessment of HER-2/neu immunohistochemical expression in breast cancer. IEEE transactions on medical imaging, 28(6), 916925.

9. Meyer, G. E. and Neto, J. C. (2008). Verification of Color Vegetation in this for automated Crop Imaging Applications. Comput. Electron. Agr, 282293.
10. Perez, A., Larranaga, P., and Inza, I. n. (2009). Bayesian Classifiers Based on Kernel Density Estimation : Flexibel Classifiers. IMT. J. Approx Reason 50, 341-362.

11. Perez, E. A., et al. (2014). HER2 testing: Current status and future directions. ELSEVIER, Cancer Treatment Reviews 40 276-284.

12. Pitas, I., and Venetanopoulos, A. N. (1990). Nonliniear Digital Filters : Principles and Applications. Kluwer Academic.

13. Ruifrok, A. C. and Johnston, D. A. (2001). Quantification of histochemical staining by color deconvolution. Anal Quant Histol, 291-299.

14. Serra, J. (1982). Image Analysis and Mathematical Morphology. Academic Press.

15. Silverman, B. W. (1986). Density Estimation for Statistics for Data Analisys. Appl. Stat. 37, 120. 